\title{
The domain of dependence fusion of two radars under distributed station-distribution
}

\author{
Chenyu Liu ${ }^{1,}$, Hongwei Zhang ${ }^{1, a}$ and Juntao $\mathrm{Ma}^{1, \text { a }}$ \\ ${ }^{1}$ Mechanical Engineering College, Shijiazhuang 050003, China; \\ a406861173@qq.com
}

\begin{abstract}
Keywords: Correlation region integration, domain of dependence, distributed Radar,Cross cumulant.
\end{abstract}

\begin{abstract}
Along with the development of modern warfare, the emergences of various kinds of low-altitude and slow-speed dim targets and sea-surface slow-speed dim targets makes the defensive radar be faced with increasingly severe tests of detecting in clutter; currently, it is difficult for a regular single radar to detect land and sea clutter in echo of low-altitude and slow-speed dim targets. Considering the relatively weak radar cross section (RCS) correlation of sea clutter and echo under different radar carrier frequencies, and the relatively strong RCS correlation of target, it is available to accumulate signals in related regions to improve signal to clutter ratio (SCR), and be conductive to detecting low-altitude and slow-speed dim targets. Based on analyzing the domain of dependence integration of two radar under adjacent station-distribution, this paper puts forward accumulation algorithm for the domain of dependence integration under distributed station-distribution in terms of two dimensional "fast-slow" time search method, and subsequently conducts a corresponding simulation experiment to testify the feasibility of the method
\end{abstract}

\section{Introduction}

Thanks to the advancement of the new system and new technology of radar, people have broken away from the restriction of merely using single radar gradually, while integrated usage of multi-radar has turned into a new radar system in the modern warfare[1]. However, the majority of present multi-radar's information fusion detects targets by signal fusion based on high levels such as decision level[2], which commonly leads to the loss of partial information in original echo signal, whilst the fusion directly based on signal levels, such as echo signal, is comparatively less[3]. Particularly, for regular radar with a narrower bandwidth, it is usually used to undertake the task of monitoring targets, and integrated usage of multi-radar therefore becomes more significance in practice. Moreover, as ocean exploration in military and civilian areas intensifies, today marine target detection has played an increasingly significant role[4]. For slow-speed targets at sea, the sea clutter in echo is so strong that single radar is very hard to make an effective detection under the condition of single frame; and the correlation time of sea clutter is so long that echo can only be accumulated during the scanning room to increase the signal to clutter ration, while prolonged accumulation frequently results in the loss of targets' slow dynamic characteristics, which bring much difficulty to the compensation of echo signal. Research shows that focusing on slow-speed targets, RCS correlation of sea clutter is relatively weaker, while RCS correlation of the target is relatively stronger in echo of different frequency band radar observation[5]. Therefore, it will be able to achieve accumulation of the domain of dependence integration for two radar echo, by calculating and accumulating cross cumulant of two radars' corresponding echo pulse envelopes, and enhance SCR which will benefit to further detection and other processes[6]. this paper build the model of two radar observing object under distributed station-distribution on the basis of process of the accumulation of the domain of dependence integration for two radar under adjacent station-distribution, as well as applying two dimensional "fast-slow" time search method to fulfill accumulation of the domain of dependence integration for bistatic radar under distributed station-distribution.

\section{Definition and nature of cross cumulant}

In accordance with statistical signal processing theory[7], the definition of K-order cumulant is: 


$$
C_{x_{1} X_{2} X_{3} \ldots x_{k}}\left(t_{1}, t_{2}, t_{3} \ldots t_{k-1}\right)=\operatorname{cum}\left[X_{1}(n), X_{2}\left(n+t_{1}\right), X_{3}\left(n+t_{2}\right) \ldots X_{k}\left(n+t_{k-1}\right)\right]
$$

Hereinto, $X_{k}(n)$ is a real-valued stochastic process, and $n=0, \pm 1, \pm 2 \ldots, \mathrm{k}=1,2,3 \ldots$, and $t_{1}, t_{2} \ldots t_{k-1}$ are time delay. It would be called as cross cumulant between stochastic processes when there is more than one stochastic process.

Equally, cross cumulant possesses nature as followed:

(1) Cross cumulant owns semi-invariability. If stochastic processes $\left\{X_{k}\right\},\left\{Y_{k}\right\}$ are mutually independent, then:

$\operatorname{cum}\left[X_{1}+Y_{1}, X_{2}+Y_{2}, X_{3}+Y_{3} \ldots X_{k}+Y_{k}\right]=\operatorname{cum}\left[X_{1}, X_{2}, X_{3} \ldots X_{k}\right]+\operatorname{cum}\left[Y_{1}, Y_{2}, Y_{3} \ldots Y_{k}\right]$

(2) If stochastic process $\left\{X_{k}\right\}$ can be divided into two groups or multi-groups of statistically independent random variables, then their K-order cumulant is 0 .

Based on two features discussed above, it is easy to find that cross cumulant will be 0 theoretically, only if one group of the random variable is independent with other random variables.

As mentioned earlier, in corresponding moment clutter echo has a weak correlation and target echo has a strong correlation, while the estimated value of the target echo cross cumulant should be greater than the estimated value of clutter echo cross cumulant, under different frequency band radar illumination. Consequently, signal to clutter ratio of signal output can be improved according to output dependence that is in favor of succeeding detection of object.

\section{Correlative fusion procedure of two distributed radar}

As mentioned earlier, in different frequency band radar illuminations, clutter echo correlation is relatively weaker, whilst target echo correlation is relatively stronger at the corresponding moment, which means that estimated value of cross cumulant of target echo should be greater than clutter echo's. As a consequence, SCR of signal output can be enhanced in line with output of correlation, and it will benefit to further detection of targets.

According to literature [6]and[8]: The precondition of accumulation of the domain of dependence integration for bistatic radar is to find the corresponding relation between echo pulse and range bin of two radars scanning the same area. At the condition of adjacent station-distribution, it is only to satisfy the antenna beam synchronous scanning because that radar separation can be neglected. Whereas, the separation distance between two radars under distributed station-distribution is increased, and the distance between target and radar cannot be neglected. Owing to exist of the obvious distance, it will cause a mismatch of pulses between two radars. Even though the orientation Position of antenna beam is amended to make the antenna realize synchronous rotation, it still cannot calculate cross cumulant directly just as adjacent station-distribution by reason of different radar positions causing that the corresponding echo pulses of two radars are not scanning the same area at the same time. Aiming at this problem, this paper proposes to achieve pulse alignment through conducting two dimensional "fast-slow" time search for two radar pulse echo, and ultimately completes the calculation of cross cumulant of the corresponding pulses.

Figure 1 is a reduced graph of two radars under distributed station-distribution. As shown in figure, Point A and Point B indicate Radar 1 and Radar 2 respectively, and Point $\mathrm{C}$ is a point target in the detection zone. Separation distance between $\mathrm{A}$ and $\mathrm{C}$ is $\mathrm{d}, \mathrm{BE}$ indicates due north, length of $\mathrm{AC}$ is $R_{1}$, distance between $\mathrm{B}$ and $\mathrm{C}$ is $R_{2}$, and the angle between $\mathrm{BC}$ and due north (BE) is $\beta$. When $\mathrm{AD}$ and $\mathrm{BE}$ indicate due north, if Radar 1and 2 anticlockwise scan, then the first step of pretreatment for bistatic radar echo under non-adjacent station-distribution is to conduct the amendment in terms of time difference of arrival when antenna passing due north just as the condition of adjacent station-distribution, which will not be repeated in this paper. After the amendment, radar beam pointing satisfies synchronous scanning (by this time antennas simultaneously passing due north and satisfying synchronous scanning). Nonetheless, at this moment echo pulses of two radars cannot reach to the precondition of calculating cross cumulant, namely echo pulses are not in the same area 
with synchronous scanning due to exist of the distance between radars, which is required to be further amended. In order to analyze conveniently, beam cut-off line is regarded as a radius and thereby to draw up the circumstance of radar antenna scanning a circle. Then, a reduced graph of the beam cut-off line in the vertical view is as followed:
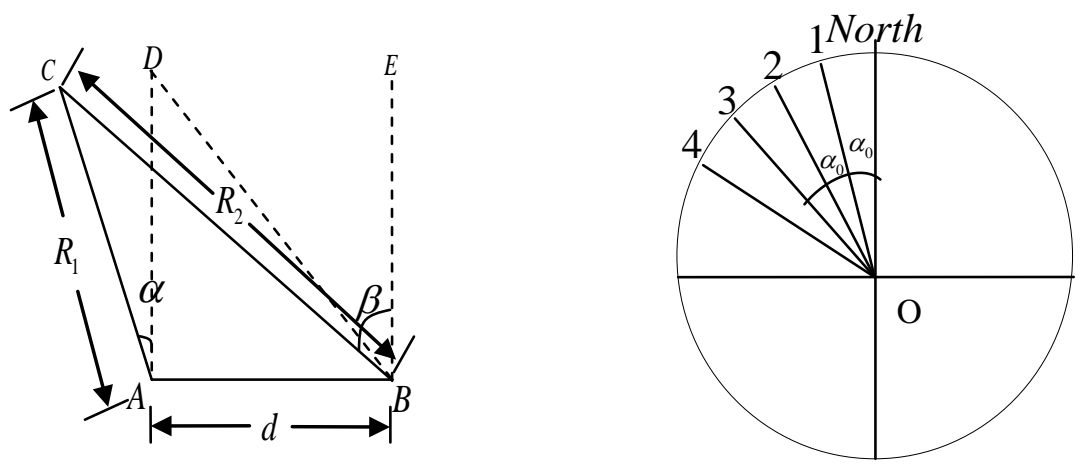

Fig. 1 simplified diagram of separate double radar observation and radar beam scanning

The general procedure is summarized as follows:

If range bin echo signal of Radar 1's random pulse in is $E c h o_{i_{1}}^{j_{1}}$, and $i_{1}, j_{1}$ there into indicate echo sequence number and range bin sequence number respectively, satisfying:

$1 \leq i_{1} \leq N_{\text {all }}, N_{\text {all }}=T_{\text {scan }} f_{r}$

$1 \leq j_{1} \leq f i x\left(\frac{c}{2 f_{r} R_{0}}\right)$

$N_{\text {all }}$ indicates the number of pulses of Radar 1 antenna scanning a circle, $T_{\text {scan }}$ indicates the time of antenna scanning a circle, $f_{r}$ indicates repetition frequency of radar pulse, $c$ indicates velocity of light, $R_{0}$ indicates the distance represented by the setting length of range bin, and fix(.) refers to round-off。

Radar 1echo pulse will be evaluated by each range bin according to the pulse sequence number, for number $j_{1}$ range bin of number $i_{1}$ echo pulse it can obtain that:

$$
\begin{aligned}
& R_{2 M_{2}}^{2}=d^{2}+\left(j_{1} R_{0}\right)^{2}-2 d j_{1} R_{0} \cos \left(i_{1} \alpha_{0}+\frac{\pi}{2}\right) \\
& \left(j_{1} R_{0}\right)^{2}=d^{2}+R_{2 M_{2}}^{2}-2 d R_{2 M_{2}} \sin \beta
\end{aligned}
$$

Then, pulse sequence number $i_{2}$ in the same area Radar 2 scanning and the corresponding range bin sequence number $j_{2}$ can be found. According to mentioned above, to divide two echo pulses of Radar1 and 2, as well as the following $(N-1)$ echo pulses into two groups respectively, Radar1 and Radar 2echo pulse group is

$$
\begin{aligned}
& E_{1\left(i_{1}\right)}^{j_{1}}=\left[\operatorname{Echo}_{1\left(i_{1}\right)}^{j_{1}}, \operatorname{Echo}_{1\left(i_{1}+1\right)}^{j_{1}}, \ldots, \operatorname{Echo}_{1\left(i_{1}+N-1\right)}^{j_{1}}\right] \\
& E_{2\left(i_{2}\right)}^{j_{2}}=\left[\operatorname{Echo}_{2\left(i_{2}\right)}^{j_{2}}, \operatorname{Echo}_{2\left(i_{2}+1\right)}^{j_{2}}, \ldots, \operatorname{Echo}_{2\left(i_{2}+N-1\right)}^{j_{2}}\right]
\end{aligned}
$$

These two groups of echo pulses are the corresponding echo of bistatic radar scanning the same area under distributed station-distribution. As previously mentioned, by calculating and accumulating the cross cumulant of these two group of echo pulses' range bin, it can obtain the number $j_{1}$ range bin of number $i_{1}$ echo pulse of Radar 1 , and output quantity of domain of dependence accumulation for the corresponding echo pulse of Radar 2 scanning the same area and the corresponding range bin under distributed station-distribution. Regarding Radar 1 as standard, the output quantity is denoted as:

$$
C_{1\left(i_{1}\right)}^{j_{1}}=\operatorname{cum}\left(E_{1\left(i_{1}\right)}^{j_{1}}, E_{2\left(i_{2}\right)}^{j_{2}}\right)
$$


For the number $i_{1}$ echo pulse of Radar 1 , by traversing all range bins of it. By repeating this process, under distributed station-distribution, it can obtain an accumulation matrix of domain of dependence Integration for each range bin of the number $i_{1}$ echo pulse of Radar 1 , and each echo pulse group when the corresponding radar scanning in the same area, which is denoted as:

$$
A_{\left(i_{1}\right)}=\left[C_{1\left(i_{1}\right)}^{1}, C_{1\left(i_{1}\right)}^{2}, \ldots, C_{1\left(i_{1}\right)}^{M}\right]
$$

If the target appears in number $i_{1}$ echo pulse of Radar 1 , then by this time the target is just scanned by the cut-off line of the radar beam. In $A$, the domain of dependence output value of range bin where the target located is relatively larger, while the output value of range bin where the target not located is relatively lower, and thereby to enable two radar under distributed station-distribution to complete accumulation matrix of domain of dependence Integration

\section{Simulation analysis}

Target is set as a point object, and its amplitude response will not change along with time. The distance between it and radar is $15.15 \mathrm{~km}$, sampling number of range bin $=50, S C R_{\text {in }}=8 d B$. According to the calculation, the target will be found in the 41th sequence number of echo pulse of Radar 1, Next, it will make a simulation of the domain of dependence integration under distributed station-distribution. With fundamental conditions of simulation unchanged, limit the number of echo pulse received by Radar 1 in the first 15 due to the restriction of simulation data, which will be used to conduct two dimensional "fast-slow" time search. According to the mentioned above, the target is non-cooperative in the real process, Moreover, each pulse of Radar 1 is required to traverse its 200 range bins, and thereby to calculate cross cumulant and complete the accumulation of the domain of dependence integration. The simulation result is as shown in figure 1 and figure 2:
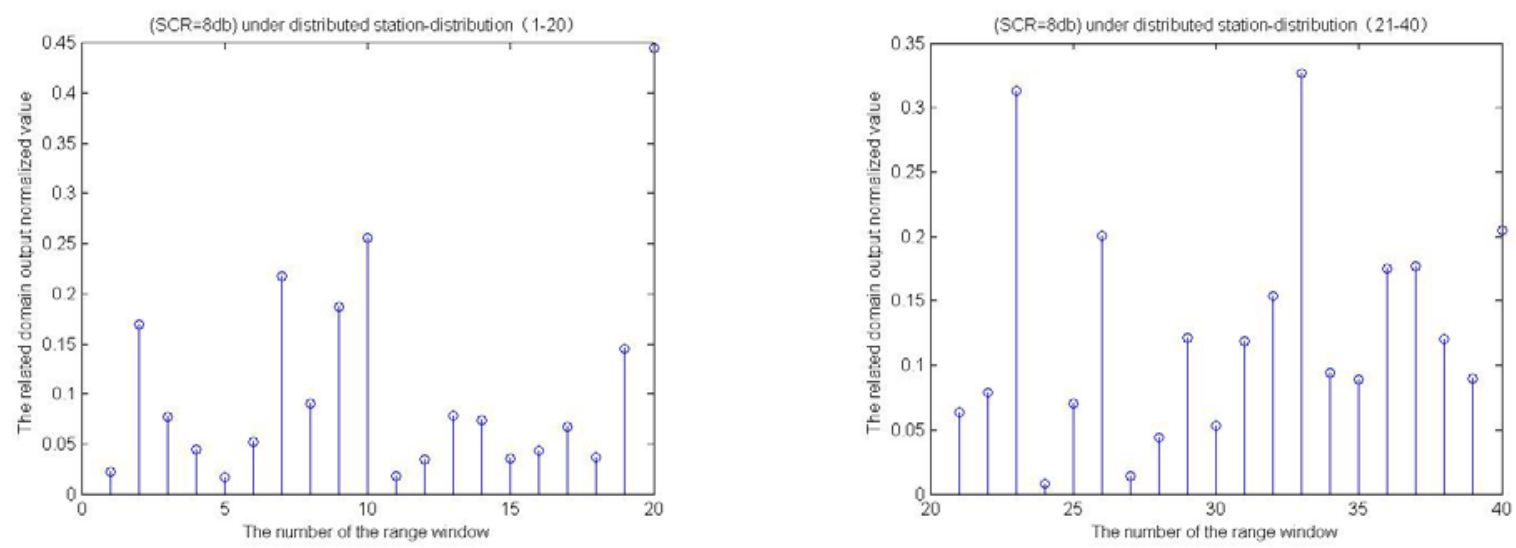

Fig. 2 Number 1-40 distance window correlation domain fusion result of second order cross cumulant after search

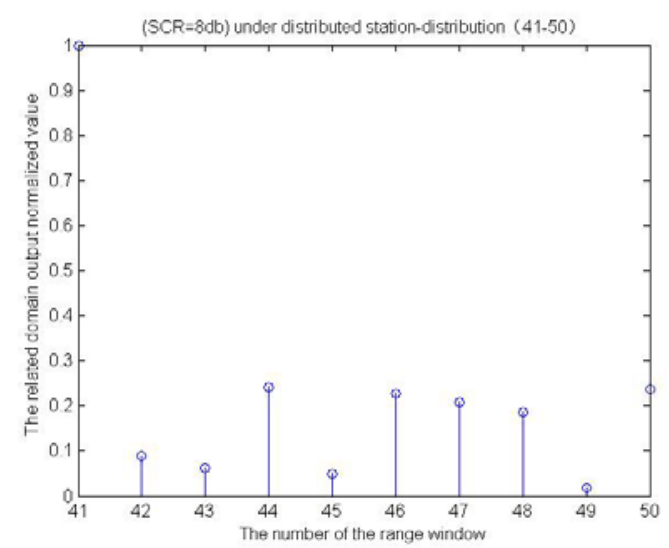

Fig. 3 Number 41-50 distance window correlation domain fusion result of second order cross cumulant after search 


\section{Summary}

For radar networking system, there is a great diversity of models for radar station-distribution so as to adapt different strategic and tactical demands. Based on two radar observing object under adjacent station-distribution, thereby to propose the accumulation algorithm for the domain of dependence integration when two radar is under distributed station-distribution, i.e. set Radar 1 as the standard, and locate echo pulse sequence number and range bin position in the same area that Radar 2 scanning by means of two dimensional "fast-slow" time search method with the purpose of completing accumulation of domain of dependence integration. Furthermore, it conducts the simulation analysis with specific echo pulse sequence number and the corresponding results of range bin, as well as the final output results of accumulation of domain of dependence integration. In general, the results are comparatively ideal, and can be used to prove the validity of the algorithm for two radar under distributed station-distribution.

\section{References}

[1]Huabang Zhu,Juan Du. “Four threats”survival and combat environment radar technology analysis [J].Aerodynamic Missile ,2005,01:61-64.

[2] You He,Xin Guan,Guohong Wang. Research progress and Prospect of multi sensor information fusion [J]. Acta Astronautica,2005,04:524-530.

[3]Cheng Wang. Study on imaging technology of radar signal layer fusion [D]. National Defense Science and Technology University,2006

[4] Chong Wu,Haifei Li,Hong Wang,Jianbin Li.The analysis of PD radar detecting low-altitude and slow-speed dim target performance [J].Science Technology and Engineering,2013,28:8492-8496.

[5]You He,Yong Huang,Xiaolong Chen. Review of radar target detection in sea clutter [J]. Modern Radar,2014,12:1-9.

[6]Cheng Wang,Weidong Hu,Xiaoyong Du,Wenxian Yu. Multi radar signal level fusion detection based on mutual accumulation[J].Signal Process,2006,04:480-485

[7]Cheng Yin,Zhiming Wu,Huaiqun Deng Application of high-order statistics in seismic exploration [J]. Progress In Geophysics,2003,03:546-550,2006,06:41-45+65

[8] Cheng Wang,Weidong Hu,Xiaoyong Du,Wenxian Yu. Application of signal level fusion in slow moving small target detection [J]. Modern Radar, 